HEALTHCARE DELIVERY

\title{
Indications for the use of bronchial thermoplasty in severe asthma
}

\begin{abstract}
K Dheda, C F N Koegelenberg, A Esmail, E Irusen, M E Wechsler, R M Niven, K F Chung, E D Bateman
Prof. Keertan Dheda is Head of the Lung Infection and Immunity Unit and the Division of Pulmonology, Department of Medicine and UCT Lung Institute, University of Cape Town and Groote Schuur Hospital, Cape Town, South Africa. Prof. Coenie Koegelenberg is a consultant in Pulmonology and Critical Care at Stellenbosch University and Tygerberg Academic Hospital, Tygerberg, Cape Town. Dr Aliasgar Esmail is a consultant pulmonologist in the Lung Infection and Immunity Unit and the Division of Pulmonology, Department of Medicine and UCT Lung Institute, UCT and Groote Schuur Hospital. Prof. Elvis Irusen is Head of the Division of Pulmonology at Stellenbosch University and Tygerberg Academic Hospital. Prof. Michael Wechsler is Co-Director of the Asthma Institute, Department of Medicine, Division of Pulmonary, Critical Care and Sleep Medicine, National Jewish Health, Denver, CO, USA. Dr Rob M Niven is a consultant chest physician and Senior Lecturer in Respiratory Medicine at the University Hospital of South Manchester NHS Foundation Trust, University of Manchester, UK. Prof. Kian Fan Chung is the Head of Experimental Studies at the National Heart and Lung Institute, Imperial College London and National Institute for Health Research, and Consultant Chest Physician at the Royal Brompton \& Harefield NHS Trust. Emeritus Prof. Eric Bateman is Director of the UCT Lung Institute and Honorary Consultant in the Division of Pulmonology, UCT and Groote Schuur Hospital.
\end{abstract}

Corresponding author: K Dheda (keertan.dheda@uct.ac.za)

Approximately $5 \%$ of the $~ 3$ million asthmatics in South Africa have severe asthma that is associated with substantial morbidity, cost, absenteeism, preventable mortality, and the requirement for costly chronic medication that may be associated with significant adverse events. There is an unmet need for alternative safer and more effective interventions for severe asthma. A recently introduced option, bronchial thermoplasty (BT), imparts radiofrequency-generated heat energy to the airways to cause regression of airway smooth muscle. The effectiveness of this technique has been confirmed in randomised control trials and is now endorsed by several international guidelines, including the Global Initiative for Asthma (GINA) guideline, the British Asthma Guideline, and the UK National Institute of Clinical Excellence (NICE) guideline. We recommend BT as a potential therapeutic intervention for severe uncontrolled asthma, provided that it is performed by an experienced pulmonologist at an accredited centre and done within the broader context of appropriate management of the disease by doctors experienced in treating difficult-to-control asthma.

S Afr Med J 2015;105(10):808-809. DOI:10.7196/SAMJnew.8208

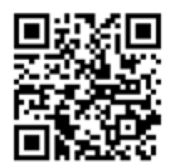

\section{The burden of severe asthma} and the unmet need

There are almost 3 million asthma sufferers in South Africa (SA), which ranks fourth highest in asthma mortality globally. In a subset of about $5 \%$ of asthma patients, the disease remains uncontrolled despite optimised therapy and good adherence. These individuals experience substantial morbidity, time off work or school, and increased mortality. Severe asthma consumes a disproportionate amount (up to $80 \%$ ) of asthma-related healthcare resources. Oral corticosteroids, alternative oral agents and biological agents such as omalizumab, when appropriate, are often required to improve or maintain control. ${ }^{[1-3]}$ These therapies are associated with substantial cost and adverse events, and efficacy remains suboptimal. Better tools are urgently needed. A procedure called bronchial thermoplasty (BT) has recently been validated in clinical trials and is now available in over 30 countries worldwide. We summarise key aspects of BT including the concept, the mechanism of action, the efficacy and safety of the procedure, and in what context it should be used in clinical practice. A more comprehensive outline of the procedure, indications and contraindications, adverse events, evidence for long-term efficacy and safety, recommendations about using BT in South Africa, and international guidelines and agencies that have endorsed the procedure was provided in a more detailed article in last month's $S A M)^{[4]}$

\section{What is bronchial thermoplasty and how does it work?}

Bronchial thermoplasty entails imparting radiofrequency-generated heat energy to medium-sized and large airways using a special catheter introduced through a bronchoscope (Fig. 1). The procedure takes approximately 45 minutes and is usually performed under conscious sedation with same-day discharge; general anaesthesia can be used in appropriate cases. Three treatment sessions 3 - 4 weeks apart are required. Animal and human studies have demonstrated that BT results in substantial regression of airway smooth muscle, ${ }^{[5,6]}$ but other mechanisms may underpin the beneficial effects, including an impact on airway wall remodelling, immune modulation and neurogenic function.

\section{Efficacy, safety and duration of benefit} The efficacy of BT has been confirmed in two feasibility studies ${ }^{[7,8]}$ and three randomised controlled trials. ${ }^{[9-11]}$ Collectively, these studies have shown that BT improves asthma control and asthma-related quality of life scores. However, most important in patients with severe asthma are reductions in exacerbations, asthma-related emergency room visits, and the need for oral corticosteroids. Two long-term prospective follow-up studies have shown that these benefits are sustained for up to 5 years. ${ }^{[12,13]}$ Three long-term studies have shown that BT is safe and not associated with any long-term adverse events such as bronchiectasis or airway stenosis. ${ }^{[12-14]}$ Short-term adverse effects of the procedure, which occur in $5-10 \%$ of cases, include precipitation of an exacerbation that may require short-term hospital admission. No pneumothorax, intensive care unit-related admissions, or fatalities have been reported so far among the over 4000 patients who have undergone the procedure. On the basis of these data, the use of BT for severe asthma has been endorsed by several international guidelines including the 2014 British Thoracic Society/ 
Scottish Intercollegiate Guidelines Network (SIGN) Asthma Guideline (British Guideline on the Management of Asthma), the 2014 UK National Institute of Clinical Excellence (NICE) and Global Initiative for Asthma (GINA) guidelines, and the 2014 European Respiratory Society/American Thoracic Society (ERS/ATS) Guidelines on the Definition, Evaluation, and Treatment of Severe Asthma.

\section{When should BT be considered?}

BT should be considered in difficult-to-treat asthma when the usual recommended measures described in treatment guidelines have been employed. ${ }^{[1-3]}$ Treatment should have been assessed and optimised by a clinician experienced in managing severe asthma, and should include the following steps: ( $i$ ) confirmation of the diagnosis of asthma; (ii) exclusion by relevant investigations of alternative diagnoses that can masquerade as asthma, such as chronic obstructive pulmonary disease, cardiac failure, bronchiectasis, vocal cord dysfunction, endobronchial sarcoidosis and tuberculosis, obstructive airway lesions and inhaled foreign bodies, among others, as potential causes of or contributors to patients' symptoms (BT is not indicated in bronchopulmonary aspergillosis); (iii) consideration and where possible correction of comorbid medical or psychosocial factors that contribute to the failure of asthma control (comorbid conditions include gastrooesophageal reflux disease, rhinosinusitis and drug allergies); (iv) an attempt at nonpharmacological measures such as removal, where possible, of contributing environmental and occupational allergens or factors that precipitate or worsen asthma; and $(v)$ finally, and importantly, optimal pharmacological measures indicated for the treatment of severe asthma (described as steps 4, 5 and 6 in different guidelines) must have been employed under supervision for at least 3 months, with careful checking and confirmation of good inhaler technique and adherence to the drugs. The treatments described on steps 4 and higher are maintenance treatment with at least medium to high doses of inhaled corticosteroid with one or more additional controller medications, which may include a long-acting beta-2agonist (LABA), a long-acting antimuscarinic (tiotropium has recently been approved in many countries for the treatment of severe asthma), ${ }^{[2]}$ slow-release theophylline, and a leukotriene modifier (montelukast). Finally, patients may have received or be taking maintenance oral corticosteroids, or have received treatment with omalizumab. ${ }^{[3]}$ Omalizumab is an injectable monoclonal antibody recently approved for use in SA for patients with severe allergic asthma. Disadvantages of maintenance oral

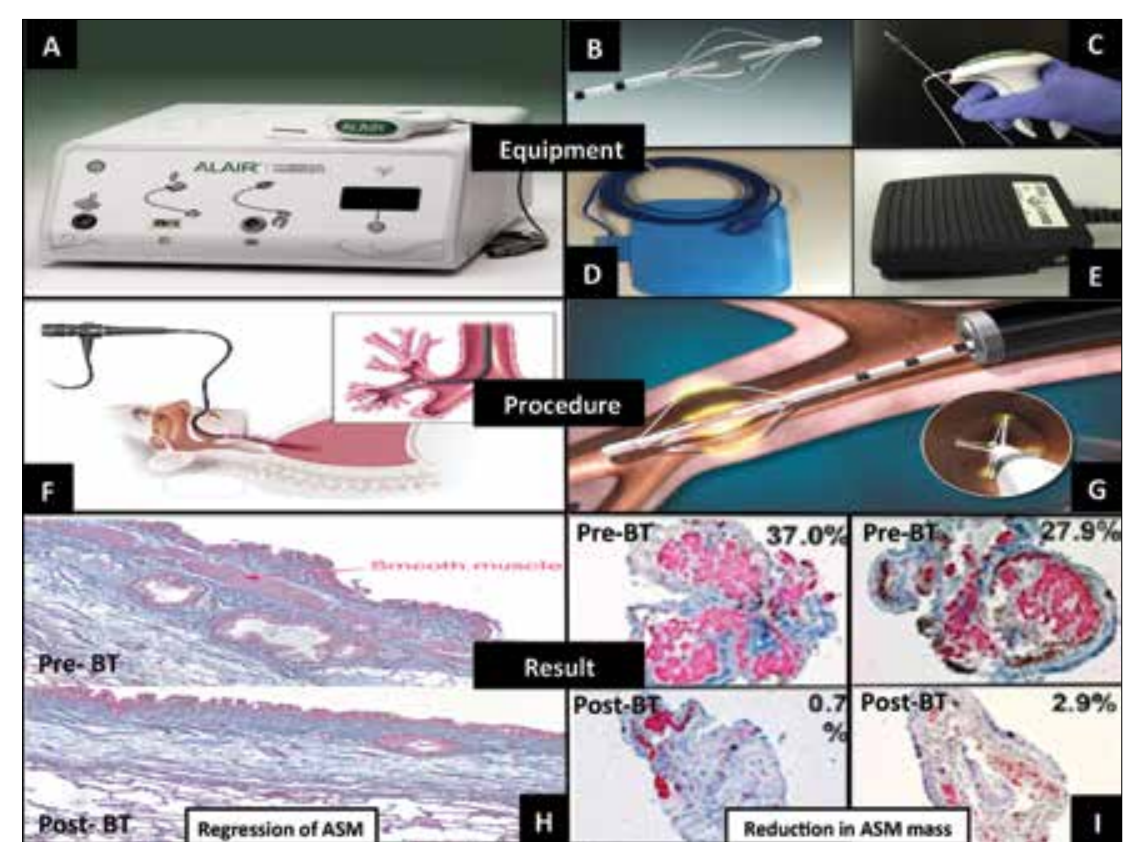

Fig. 1. Outline of the equipment, the BT procedure, and effects on airway smooth muscle. A: Alair radiofrequency controller; B: Alair BT catheter tip (basket electrode) with the $5 \mathrm{~mm}$ spacer markings guiding sequential activations; $C$ : Handle grip controlling expansion of the catheter tip; D: Gel-type return electrode to complete the circuit; E: Foot pedal triggering delivery of the radio frequency-mediated thermal energy; F: BT procedure performed using a flexible fibreoptic bronchoscope; G: Catheter in the bronchus delivering controlled radiofrequency thermal energy; H: Histological sections of dog bronchial wall before (top) and 12 weeks after BT (bottom), showing reduction in airway smooth muscle and preserved integrity of the epithelium, mucous glands and subepithelial tissue; ${ }^{[6]} \mathrm{I}$ : Biopsies before and after BT in the lower lobes of two patients, showing reduction in airway smooth-muscle mass (the percentage of airway smooth-muscle surface area/total biopsy area) is shown numerically in the sub-figure. From www.btforasthma.com, reproduced with permission.

corticosteroids are their short- and long-term side-effects, while the use of omalizumab is limited by cost, lack of efficacy in a significant proportion of patients, and the necessity for indefinite use.

\section{Conclusion}

BT is indicated in patients with severe asthma who remain uncontrolled despite optimal work-up and medical therapy. BT should only be performed by an experienced pulmonologist at an accredited centre, preferably as part of a national or international registry or a prospective study, so that long-term safety and efficacy can be evaluated. Performed under these strict conditions and according to these recommendations, $\mathrm{BT}$ is a useful addition to the treatments available for this debilitating and life-threatening condition.

1. Chung KF Wenzel SE Brozek JL et al Intenatonat Chung KF, Wenzel SE, Brozek JL, et al. International ERS/
ATS guidelines on definition, evaluation and treatment of severe asthma. Eur Respir J 2014;43(2):343-373. [http://dx.do org/10.1183/09031936.00202013

2. Global Strategy for Asthma Management and Prevention. Global Initiative for Asthma (GINA) 2015. http://www.ginasthma.org/ (accessed 29 July 2015)

3. SIGN. British Guideline on the Management of Asthma. British Thoracic Society, Scottish Intercollegiate Guidelines Network, 2014. http://www.sign.acuk/pdf/SIGN141.pdf (accessed 6 September 2015). http://www.sign.ac.. for the use of bronchial thermoplasty in the management of severe asthma. S Afr Med J 2015;105(9):726-732. [http://dx.doi org/10.7196/SAMInew.8207]

5. Danek CJ, Lombard CM, Dungworth DL, et al. Reduction in airway hyperresponsiveness to methacholine by the application of RF energy in dogs. J Appl Physiol 2004;97(5):1946-1953. [http://dx.doi.org/10.1152/japplphysiol.01282.2003]

6. Pretolani M, Dombret MC, Thabut G, et al. Reduction of airway smooth muscle mass by bronchial thermoplasty in patients with severe asthma. Am J Respir Crit Care Med 2014;190(12):14521454. [http://dx.doi.org/10.1164/rccm.201407-1374LE]

7. Cox G, Miller JD, McWilliams A, Fitzgerald JM, Lam S. Bronchial thermoplasty for asthma. Am J Respir Crit Care Med 2006;173(9):965969. [http://dx.doi.org/10.1164/rccm.200507-1162OC]

8. Miller JD, Cox G, Vincic L, Lombard CM, Loomas BE, Danek CJ. A prospective feasibility study of bronchial thermoplasty in C). A prospective feasibility study of bronchial thermoplasty in
the human airway. Chest 2005;127(6):1999-2006. [http://dx.doi org/10.1378/chest.127.6.1999]

9. Castro M, Rubin AS, Laviolette M, et al. Effectiveness and safety 9. Castro M, Rubin AS, Laviolette M, et al. Effectiveness and safety
of bronchial thermoplasty in the treatment of severe asthma: A multicenter, randomized, double-blind, sham-controlled clinical trial. Am J Respir Crit Care Med 2010;181(2):116-124. [http://dx.doi.org/10.1164/rccm.200903-0354OC]

0. Cox G, Thomson NC, Rubin AS, et al. Asthma control during the year after bronchial thermoplasty. N Engl J Med 2007;356(13):13271337. [http://dx.doi.org/10.1056/NEJMoa064707]

1. Pavord ID, Cox G, Thomson NC, et al. Safety and efficacy of bronchial thermoplasty in symptomatic, severe asthma. Am J Respir Crit Care Med 2007;176(12):1185-1191. [http://dx.doi. org/10.1016/j.anai.2013.05.002]

12. Pavord ID, Thomson NC, Niven RM, et al. Safety of bronchial thermoplasty in patients with severe refractory asthma. Ann Allergy Asthma Immunol 2013;111(5):402-407. [http://dx.doi. Allergy Asthma Immunol 2013; 11
org/10.1016/j.anai.2013.05.002]

13. Wechsler ME, Laviolette M, Rubin AS, et al. Bronchial thermoplasty: Long-term safety and effectiveness in patients with severe persistent asthma. J Allergy Clin Immunol 2013;132(6):1295-302. [http:/ dx.doi.org/10.1016/j.jaci.2013.08.009]

4. Thomson NC, Rubin AS, Niven RM, et al. Long-term (5 year) safety of bronchial thermoplasty: Asthma Intervention Research (AIR) trial. BMC Pulm Med 2011;11:8. [http://dx.doi. org/10.1186/1471-2466-11-8]

Accepted 31 July 2017. 